\title{
Robust wireless sensor network performance analysis
}

\author{
K. Martinez, P. Basford \\ Electronics and Computer Science, University of Southampton \\ Southampton, UK \\ $\{\mathrm{km}$, pjb08r $\} @$ ecs.soton.ac.uk
}

\begin{abstract}
We show that a wireless sensor network system can be designed specifically for a set of complex deployment requirements and constraints. Among the design issues tackled are: low power design which copes with arctic winters and adaptive behaviour to cope with communications breakdowns. These techniques were implemented in a WSN deployment in Iceland in 2008 by the Glacsweb team. This paper shows how this has allowed base stations to survive the winter for the first time.
\end{abstract}

Rather than scaling up the power sources to cope, the systems scale-back their activities, especially communications and dGPS sensing. Similarly instead of overdesigning the radio networks, disconnection periods were managed using large multi-level buffers. The overall success was increased by techniques introduced after the main deployment in 2008. The system is comprised of eight subglacial sensor nodes, connected to a base station on the glacier and a fixed reference station on the mountainside.

\section{INTRODUCTION}

Deploying wireless sensor networks in and on glaciers is an engineering challenge and creates completely new data streams for glaciologists. These data streams are currently being used to investigate stick-slip behave as described in $[1,2]$, and have previously been used to examine till behaviour [3]. Previous work by the Glacsweb team has tackled hardware design [4], implementing easily maintained code on the base stations [5,6] and analysing the subglacial node data [7]. Despite the progress that is examined in these previous papers, continual base station operation through the winter has not been possible until a variety of additional power management features were implemented.

The system is comprised of two main types of sensing node. Probes which are embedded inside the Vatnajökull ice sheet $(80 \mathrm{~m}$ deep in this deployment) which record; temperature, pressure, strain, conductivity, pitch/roll orientation and light reflectivity. These probes transmit the data once a day to the surface station via a $173.250 \mathrm{MHz}$ radio link. A base station on the ice surface acts as a data relay and also takes GPS readings in coordination with a similar system in a fixed location $1 \mathrm{~km}$ away, known as the reference station. The two surface nodes are significantly more complex than

Currently funded by The Leverhulme Trust the probes, in that they have to manage the external communication off the mountain using GPRS. Due to their high power requirements they also require adaptive power management. However, all the communications and power generation in these systems are unreliable due to the harsh conditions in which they are deployed.

The surface stations use differential GPS (dGPS) which allows positioning to millimetre accuracy, however this accuracy comes at a cost long recording periods with a power draw of 3.6W.This power draw needs to be carefully managed in order for the system to survive within the power limitations of the system. The other restriction on the dGPS is the data is most useful when it is available for two stations, one whose location is previously fixed, for the same time period. In this deployment the fixed location is a café $1 \mathrm{~km}$ away from the surface system and they are not radio linked, meaning communication to determine power state has to go via Southampton

This paper examines the compromises made and the strategies in place to maximise data flow within the environmental constraints.

\section{POWER LIMITATIONS}

In order to supply power for the base station 36Ah of lead acid batteries are used, however, on their own this is not enough to sustain the number of readings that the end users of the system would like. These batteries are supplemented by incoming energy from various sources; for the station on the Ice a $10 \mathrm{~W}$ solar panel, and a $50 \mathrm{~W}$ wind generator are used. Rather than predicting the unpredictable incoming energy the system reacts to its energy levels (using battery voltage as a proxy). For the reference station a $10 \mathrm{~W}$ solar panel and a mains feed from the café (only powered June - September) are used. As the recharge sources for each node are different the available energy reserves will not necessarily match.

In order to maximise the data output of the system the sensing tasks will back off when power is more limited. The priorities for the system can be set by the researchers upon deployment and if necessary the system can be remotely reconfigured.

Four main states were defined: fast (3), daily (2) and power saving (1), minimal (0), full details of what these states entail can be found in [5]. These were triggered by simple 
thresholds on daily average battery voltage. This original system design was outlined in [4], however at that point it had not been deployed for long in the field. It is now possible to show results from the field trial to analyse its performance.

The dGPS receiver has a high current draw, however, this is not the only way in which its use consumes power. The GPS data is on average $45 \mathrm{kB} / \mathrm{min}$ ( $33 \mathrm{kB} /$ min compressed), and the longer the reading the better. The GPS serial port to the ARM runs at approx. $38 \mathrm{kbit} / \mathrm{s}$. During this download perios the GPS unit has the same power draw as the initial recording of it. This means the base station can be occupied for minutes downloading a day of readings. The total energy use of recording and downloading the data to the gumstix can be described by

$$
\text { Energy }=\mathrm{T}_{\text {recording }} * \mathrm{P}_{\text {grps }}+\mathrm{T}_{\text {download }} *\left(\mathrm{P}_{\text {grps }}+\mathrm{P}_{\text {gum }}\right) \text {. }
$$

When in the fast power state this equates to 14 kJoule per day, and in state 2 it is $1.1 \mathrm{~kJ}$ oule per day, so this shows that a massive energy saving can be gained by backing off the GPS recordings. There is also another stage of the process of taking GPS readings which uses power and that is sending data off the glacier. Whereas the daily debug and probe information is about $4 \mathrm{kB}$ as explained the GPS data is vastly more. Unfortunately it is not possible to calculate the energy used by the transmission as those times are not recorded. So clearly backing off from GPS and communications was the best way to save power, while following the user's prioritisation of the probes. In the "minimal" power state even long range communications are stopped in favour of the subglacial sensors. By stopping communications during energy poor periods it places a heavier load on the system when the higher power state is triggered. In theory this can cause flapping between states, in practice however the amount of data gathered in the minimal power state did not cause this.

\section{COMMUNICATION LiMitATIONS}

The system is deployed in a harsh environment in which all communications are unreliable. From past deployments we know that the sensor nodes embedded inside the glacier experience long periods of radio disconnection, so they use a large EEPROM buffer $(64 \mathrm{kB})$ to store sensor readings which can be sent later. Similarly the long range communications off the mountainside can be badly affected by weather so the surface stations buffer data (in compact flash). They adapt to the lossy communications by sending all buffered data once links are re-established. There have been various causes for periods of no communication; the weather is a major factor,

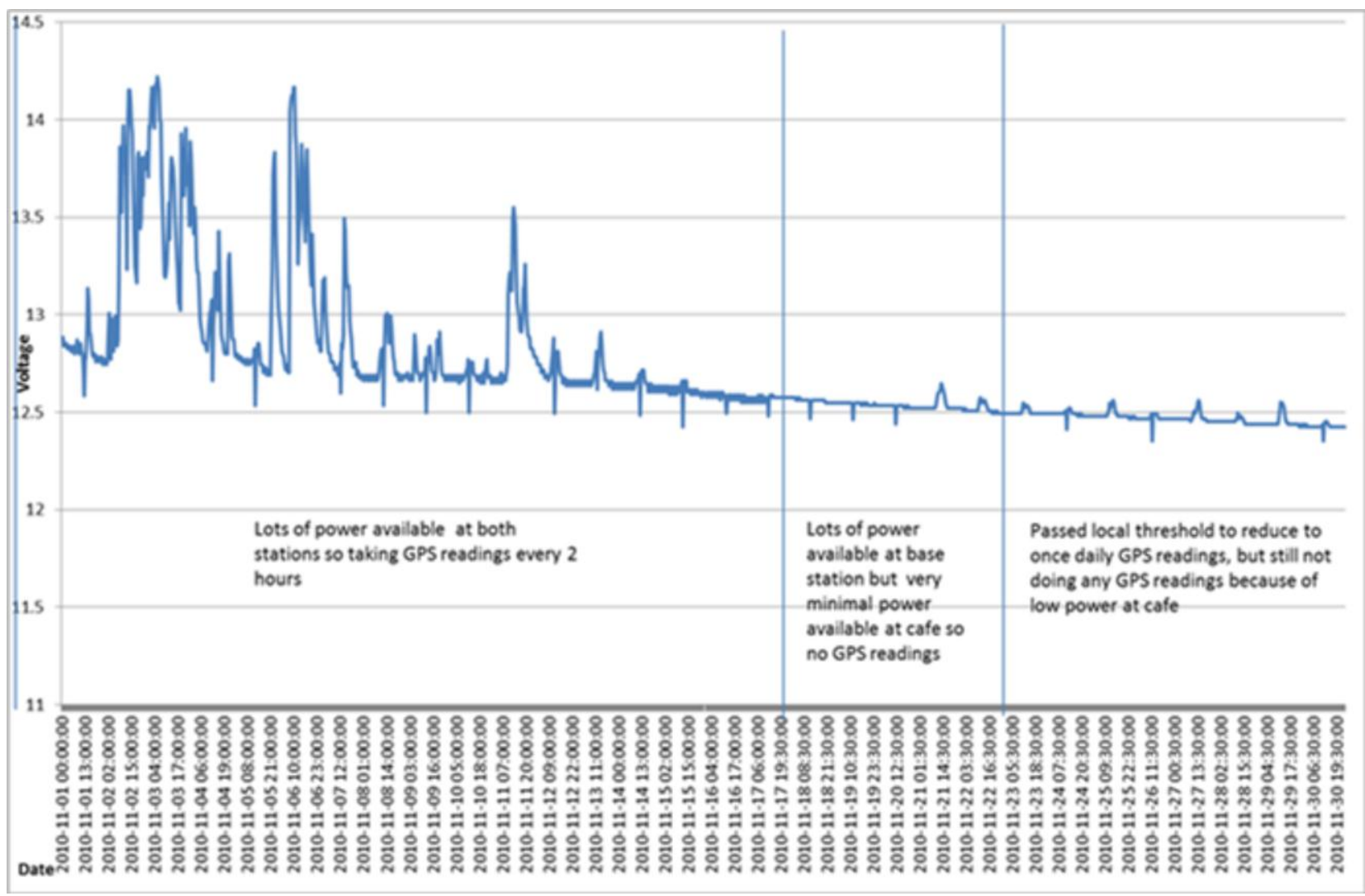

Figure 1. Base station battery voltage over time, during the winter-onset 


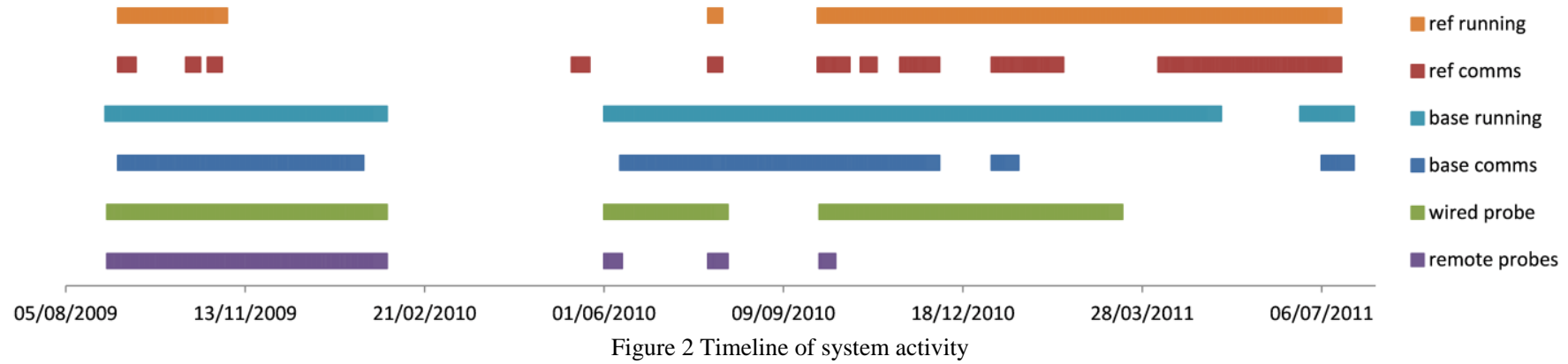

however other technological changes outside of our control have also caused issues.

\section{RESULTS}

The introduction of a system to back-off tasks depending on the battery voltage led to a longevity increase in the base stations while increasing sensor data availability during high charge periods.

The link between the power states of the two surface stations is shown in Fig. 1 where in mid-November 2010 the base station's power state was switched down to minimal due to the reference stations level dropping. Then in the final third of the chart the base station also drops its state to minimal. In the first section of the graph the spikes shown are caused by the bursty nature of the incoming power. The dips in the battery voltage coincide with the periods during which the Gumstix is power.

Fig. 2 shows the improvements in uptime of the main systems between 2009 and 2011. Whilst the system was originally deployed in 2008 it crashed in October and was only recovered by manual intervention in summer 2009, it was also then that the reference station was deployed and the power synchronisation was started.

Fig 2 also shows that despite both surface stations have the same battery reserves and being in the same power state the reference station was able to run uninterrupted through winter 2011 whereas the base station was not; this is caused by the additional power demand of the probe communications on the system. However, since the loss of communication with the wired probe this difference has been eliminated as there is no link to the rest of the probes this job cannot be attempted.

The intermittent nature of the communications with the probes is highlighted in Fig. 2 it shows that during long periods when the base was running and the wired probe was accessible there were periods without communications.

The break in communication over September 2010 was caused by a failed wire probe, and it can be seen that as soon as this was replaced data was able to flow again, at which point a couple of months of data was downloaded.

Despite the longs breaks in communication it has been possible to gather data across a two year period.

Part of the longevity of the probes can be ascribed to the radio transmission policy. The new policy which has been tried during the deployment is to send all the data that has been gathered in one long burst. Once all the data has been received by the base station it checks to see if any packets are missing based on the sequence numbers. If a packet it missing then it is requested again from the probe, once all the data has been received then the data is erased by resetting the memory pointers. This reduces the amount of time that the radio modules have to wait in RX mode, based on a $5 \%$ retry rate this leads to a $30 \%$ energy saving.

\section{FUTURE WORK}

The system being deployed in the summer of 2011 will refine the techniques used but also introduce even more power hungry communications in the form of amplified WiFi for long range communications and some sensors which will run continuously. These changes in the system mean that the backing down of sensing tasks is even more important than before. At the moment very little is known about how much power was provided by each of the charging sources, so in order to improve future reliability the current provided by each source will be measured giving a better understanding of the power situation, and opening the possibility for future power modeling.

\section{CONCLUSIONS}

This paper has shown that the power saving measures introduced into the Glacsweb sensor network have enabled the system to survive longer than previously possible. This has been achieved by backing off the high power drain tasks when the energy levels (measured using battery voltages) are low. Further savings have been made by not using 
acknowledgments for each packet that has been received by the base station, but instead requesting that the missing packets are resent after the bulk of the transmission has been completed.

It has also shown that when allowed for periods of disconnection can be recovered from. This work can also be extended by monitoring the incoming power available and so predicting future power availability and can also be applied to more power intensive devices, both these extensions will be started in an upcoming deployment.

\section{ACKNOWLEDGMENT}

The authors would like to thank Prof. J.K. Hart, Geography and the rest of the 2008/9 team: Dr. Kathryn Rose (now British Antarctic survey), Joshua Ellul (now Imperial College), Richard Clarke, Jeff Gough, Robert Spanton, Tom Bennellick, Stuart Rimmer and James Cheshire.

\section{REFERENCES}

[1] Bahr D. B. and Rundle, J. B.: Stick-slip statistical mechanics at the bed of a glacier. Geophysical Research Letters, vol. 23 (16), pp. 2073-2076. (1996).

[2] Fischer, U. H. \& Clarke, G. K. C.: Stick-slip sliding behaviour at the base of a glacier. Ann. Glaciol. Vol. 24,pp 390-396, (1997)
[3] Hart, J. K., Rose, K. C. and Martinez, K. (2011) Subglacial till behaviour derived from in situ wireless multi-sensor subglacial probes: Rheology, hydro-mechanical interactions and till formation. Quaternary Science Reviews, 30 . pp. 234-247.

[4] K. Martinez, J. K. Hart, and R. Ong, Deploying a Wireless Sensor Network in Iceland. Lecture Notes in Computer Science, Proc. Geosensor Networks, 5659, pp. 131-137, 2009

[5] K. Martinez, P. Basford, J. Ellul, and R. Clarke, Field deployment of low power high performance nodes. In: The Third International Workshop on Sensor Networks (SN 2010), 2010

[6] K. Martinez, P. Basford, J. Ellul. and R, Spanton (2009) Gumsense - a high power low power sensor node. In: 6th European Conference on Wireless Sensor Networks.

[7] J. K., Hart, K. C Rose, Waller, R. I., D. VaughanHirsch, and K. Martinez, Assessing the catastrophic break up of Briksdalsbreen, Norway, associated with rapid climate change. Journal of the Glaciology Society, 168. pp. 673-688. 2011 